$R M x A C, \mathbf{5 3}, 47-49(2021)$

(C) 2021: Instituto de Astronomía, Universidad Nacional Autónoma de México

https://doi.org/10.22201/ia.14052059p.2021.53.12

\title{
OBSERVATORIO EL SAUCE: HOSTING ROBOTIC SCIENCE IN CHILE
}

\author{
S. Ropert ${ }^{1}$, R. Rojas ${ }^{1}$, V. Suc ${ }^{1,2}$, A. Zapata ${ }^{1,3}$, J. Fertil ${ }^{1}$, and A. Jordan ${ }^{1,2}$ \\ RESUMEN
}

Observatorio El Sauce es un observatorio robótico remoto, ubicado en el norte de Chile, que ha sido diseñado para el hosting de telescopios de tamaño pequeño y mediano (diámetro $\lesssim 1 \mathrm{~m}$ ). Actualmente cuenta con telescopios utilizados con propósitos científicos, de astrofotografía y privados. Los telescopios en El Sauce han estado contribuyendo regularmente a follow-up de objetos de interés de TESS, y han paticipado en follow-up de eventos de ondas gravitacionales. En este texto describimos el observatorio, su infraestructura, y futuras instalaciones científicas que se emplazarán en el observatorio, tales como un nodo del proyecto ATLAS.

\section{ABSTRACT}

The "El Sauce" Observatory is a robotic remote observatory in Chile designed for hosting medium and small sized telescopes ( $\lesssim 1 \mathrm{~m}$ diameter). It currently hosts telescopes used for astro-photography, private and scientific purposes. Telescopes at El Sauce have been contributing regularly to follow-up of TESS objects of interests, and have participated in follow-up of gravitational wave events. In this work we describe the observatory, its infrastructure, and future scientific facilities that are planning to install at "El Sauce" such as a node of the ATLAS project.

Key Words: telescopes

\section{A ROBOTIC OBSERVATORY}

The "El Sauce" Observatory is a robotic remote observatory in Chile designed for hosting medium and small sized telescopes (Up to $1 \mathrm{~m}$ diameter). It currently hosts telescopes used for astrophotography, private and scientific purposes. Telescopes at El Sauce have been contributing regularly to followup of TESS objects of interests and have participated in follow-up of gravitational wave events.

\section{THE SITE}

The observatory is located in Valle del Rio Hurtado, $20 \mathrm{~km}$ away from Gemini South, and about 60 $\mathrm{km}$ away from the first small city. The observatory is situated on an isolated mountain $1600 \mathrm{~m}$ above sea level, giving ideal seeing conditions and a clear horizon.

\subsection{Light Pollution}

The Observatory is $60 \mathrm{kms}$ away from the first small city, this makes our sky one of the darkest in the world. Recent measurements classify our sky as a Class 1 Sky in the Bortle scale. On clear nights our SQM monitor measures an SQM close to 22. The site is endowed with an average of 300 clear nights a year in extemely dry conditions, a must for astronomical observing.

\footnotetext{
${ }^{1}$ Obstech, www.obstech.net.

${ }^{2}$ Universidad Adolfo Ibañez, Santiago, Chile.

${ }^{3}$ Pontificia Universidad Católica de Chile, Santiago, Chile.
}

\subsection{Meteorological Conditions}

The main characteristic of the observatory's meteorological conditions are listed below.

- Average of 300 clear nights per year.

- Typical seeing around 1"

- SQM in moonless clear nights close to 22

- Average Humidity of 40

- Historic min and max temperatures: $-4^{\circ} \mathrm{C}$ and $35^{\circ} \mathrm{C}$

\subsection{Services}

The observatory telescope hosting includes many services in order to secure a reliable remote operation without the need to travel to the site.

- All basic maintenance technical support is included in our standard service

- $24 / 7$ protection and daily checks on site

- Periodic mirror cleaning with $\mathrm{CO} 2$

- Debugging: Our engineers can help you locating and solving your problems 


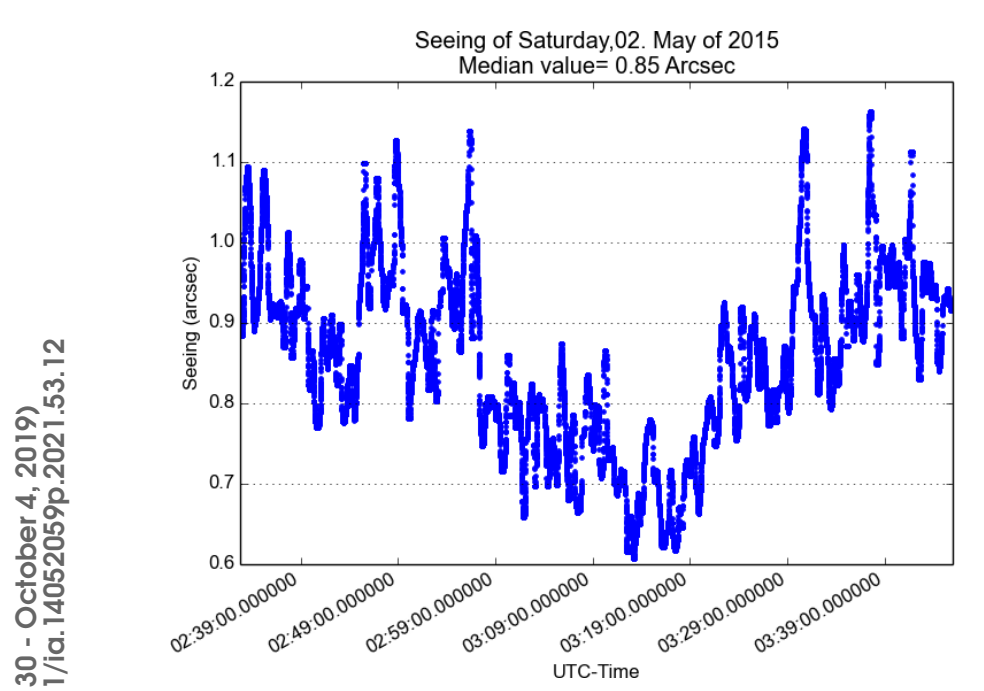

Fig. 1. Seeing Measurements using a Dimm Monitor.

TABLE 1

\section{AMOUNT OF TELESCOPES}

\begin{tabular}{lr}
\hline Use & Amount \\
\hline Astrophotography & 14 \\
Science \& R\&D & 5 \\
SSA & 18 \\
\hline
\end{tabular}

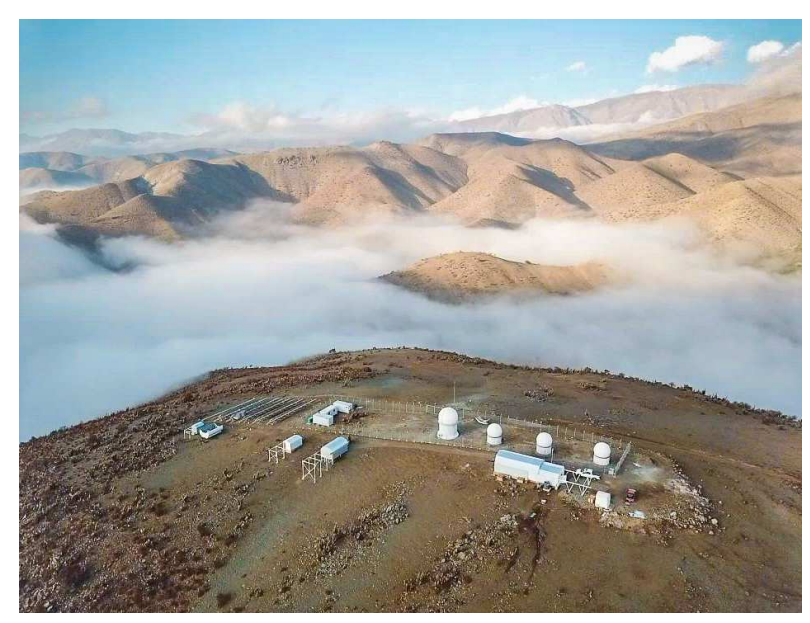

Fig. 2. Aerial view.

\subsection{Facilites}

Currently in the observatory there are 5 roll-off sheds, one clamshell shed and 4 domes, with different capacities. In total we have 37 working telescopes. During the first half of 2020, we expect to install 9 more telescopes in our roll-off sheds, and 2 dedicated domes for scientific research. The amount of telescopes per use is listed in table 1.

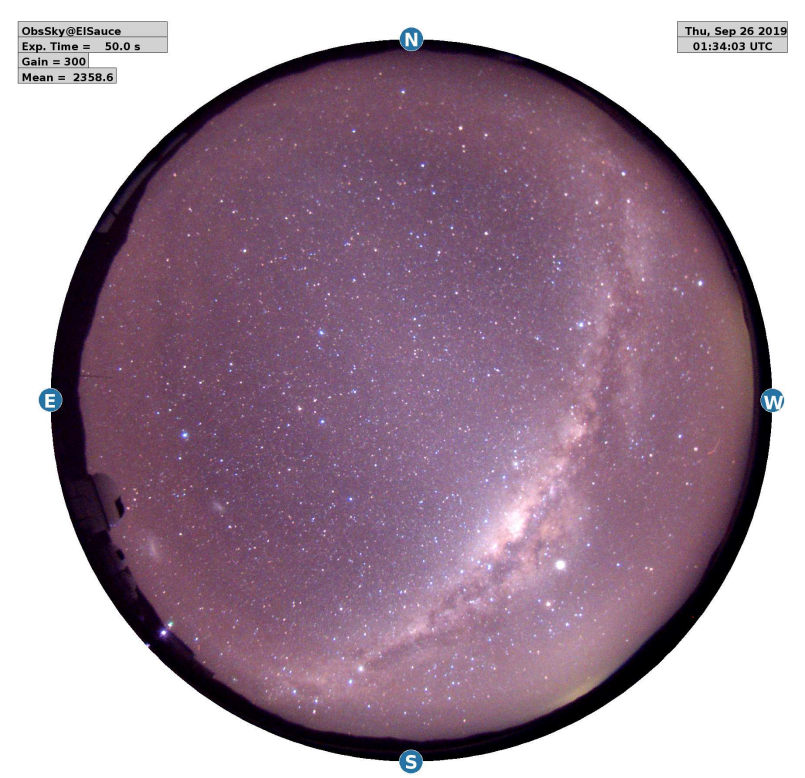

Fig. 3. Image taken by our on site All Sky camera.

The "El Sauce" Observatory is far from reaching its full capacity, there's plenty of space for new projects. A new building with capacity for 13 new telescopes is planned to be constructed during 2020. The main characteristics of the observatory are the following:

Power: $20 \mathrm{~kW}$ PV power plant and a $20 \mathrm{~kW}$ back-up diesel generator.

Internet: $100 \mathrm{Mbit} / \mathrm{s}$ symmetric fiber link + $50 \mathrm{Mbit} / \mathrm{s}$ radio link $+4 \mathrm{G}$ backup.

Telemetry: Weather Station, On site all-sky camera, SQM meter.

Remote Access: Several cameras and sensors give access to the observatory status in real time.

\section{OBSERVATION INITIATIVES}

Observatorio El Sauce currently hosts some private and public Scientific initiatives for a diversity of purposes.

\subsection{Scientific Projects}

Below we list the current scientific projects that take place in the observatory:

Phil Evans: TESS Follow-up (participated so far in the discovery of TOI150b, TOI163b, HATS-71b,LHS $3844 \mathrm{~b})$

MAS500: SNe and follow-up

Nick Dunkel: Asteroid follow-up

Chacana: Allsky Camera network for fireball detection and retrieval

Chilescope's RC 1 meter: Transient follow-up, including GW events- 


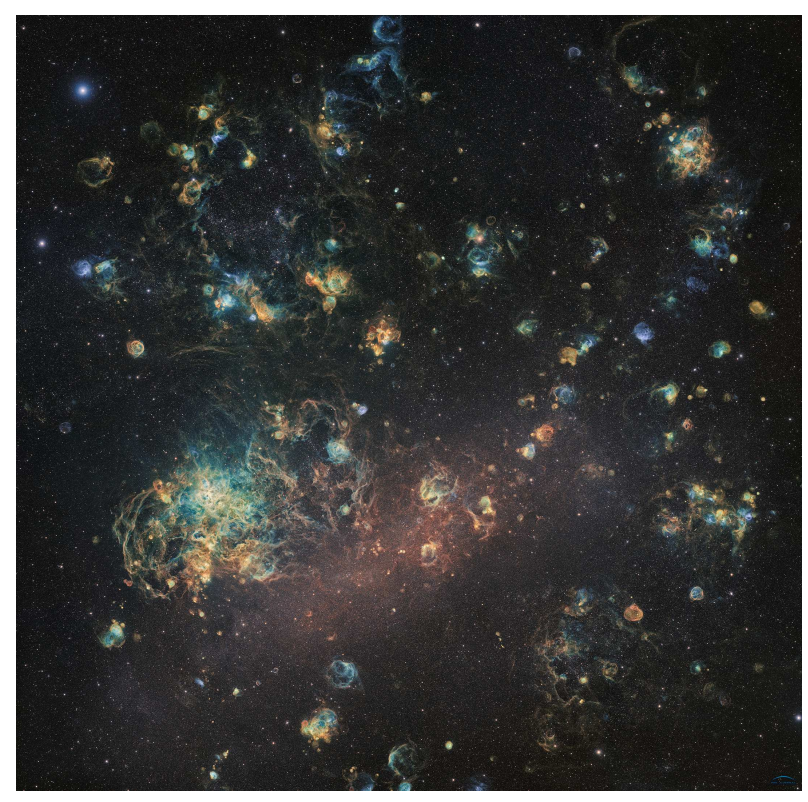

Fig. 4. Large Magellanic Cloud taken by Ciel Austral.

\subsubsection{Upcoming Projects}

In 2020 additional scientific projects will be installed in the observatory:
ATLAS: (Asteroid Terrestrial-impact Last Alert System) A 0.5m telescope part of the southern hemisphere extension of ATLAS. PI: John Tonry.

Fesenkov Astrophysical Institute: $1 \mathrm{~m}$ telescope. PI: Maxim Krugov.

\subsection{Astrophotography}

Several world renowned astrophotographers are taking advantage of our sky conditions to bring their hobby to a professional level. The seeing conditions allow our observers to make high quality images.

Figure 4 shows a mosaic of the Large Magallanic Cloud taken by the CielAustral team. With 1060 hours, it is the one of the longest exposure ever taken by amateurs.

\section{OBSTECH: DEVELOPING TECHNOLOGY FOR ASTRONOMY}

Obstech is a company formed by astronomers and engineers. Besides developing the "El Sauce" Observatory, it has been involved in a diversity of projects with different professional observatories such as La Silla (Ropert 2016), Las Campanas and Gemini South.

\section{REFERENCES}

Ropert, S., Suc, V., Jordán, A., et al. 2016, SPIE, 9912E, $4 \mathrm{WR}$ 